\title{
¿Un patio de contenedores, en el oriente antioqueño?
}

\section{¿A container yard, in the oriente antioqueño?}

\author{
Silvia Liliana Ceballos - Ramírez ${ }^{1}$, Érica Yaneth Guisao - Giraldo² \\ ${ }^{1}$ Grupo de Investigación FACEA, Universidad Católica de Oriente, Colombia, \\ ORCID: https://orcid.org/0000-0001-8733-4967, E-mail: Lceballos@uco.edu.co \\ ${ }^{2}$ Grupo de investigación en Negocios y Relaciones Internacionales, Universidad de Medellín, Colombia, \\ ORCID: https://orcid.org/0000-0002-1535-8318, E-mail: eyguisao@udem.edu.co \\ Cómo citar: Ceballos - Ramírez, S. L., \& Guisao - Giraldo, Érica Y. (2020). ¿Un patio de contenedores, en el Oriente \\ Antioqueño? Revista Científica Profundidad Construyendo Futuro, 13(13), 62-69. \\ https://doi.org/10.22463/24221783.2659
}

Recibido: 03 de marzo de 2020 / Aprobado: 15 de mayo de 2020

\begin{abstract}
Resumen
La utilización del contenedor desde la década de los cincuenta, ha generado cambios en la movilización de carga y, como resultado de los viajes de buques portacontenedores empieza la construcción de patios de contenedores o puertos secos, los cuales cobran importancia en los procesos logísticos. El propósito de este escrito es evidenciar, si un puerto seco en el Oriente Antioqueño, subregión Altiplano, aportaría a la eficiencia de los procesos de comercio exterior. La investigación fue mixta, concluyente y analítica; se realizaron entrevistas a tres (3) patios de contenedores asentadas en el departamento de Antioquia y, se hicieron encuestas a 63 empresas asentadas en la región que realizan operaciones de importación, exportación o ambas. Se resalta que los empresarios consideran que la apertura de un patio permitirá disminuir costos y tiempos de operación y poder negociar mejores tarifas con las navieras y empresas transportadoras terrestres.

Palabras clave: Carga, Empresas, Oriente Antioqueño, Patio de Contenedores, Puerto Seco.

Abstract

The use of the cargo container since the decade of fifties has generated changes in the mobilization of the load. As a result of the trips of the container ships, the construction of container yards or dry ports begins, which gain importance every day in the logistics processes. The purpose of this script is to show whether a dry port in Eastern Antioquia in the Altiplano subregion, would contribute to the efficiency of foreign trade processes. The investigation type was mixed, conclusive and analytical; Interviews were managed with three (3) container yards located in the department of Antioquia, and surveys were conducted with 63 companies located in the region that carry out import, export or both operations. It is highlighted that entrepreneurs are considering that the opening of a yard could reduce costs and operating times and be able to negotiate better rates with shipping companies and land transport companies.
\end{abstract}

Keyword: Load, Companies, Oriente Antioqueño, container area, dry port.

*Autor para correspondencia.

Correo electrónico: Lceballos@uco.edu.co

La revisión por pares es responsabilidad de la Universidad Francisco de Paula Santander Ocaña

Artículo bajo licencia CC BY-NC (https://creativecommons.org/licenses/by-nc-nd/4.0/) 


\section{Introducción}

Las operaciones de importación y exportación permiten procesos de intercambio entre los diferentes países, en donde la manipulación de la carga requiere de técnicas ágiles para que las empresas sean más competitivas; es por eso, que el movimiento de contenedores ha venido cobrando importancia al pasar del manejo de carga suelta a contenedorizada, que con la ayuda de reglas estandarizadas que pueden replicar en cualquier lugar.

Cipoletta (2010, citado en el Ministerio de Transporte, 2014), señala que los patios de contenedores se:

Deben concebir dentro del entorno sistémico de la logística de comercio Exterior, lo que implica de un análisis que los contemple como parte general de las cadenas logísticas de abastecimiento, almacenamiento y distribución, y no como eslabones independientes, desarticulados y aislados de los procesos de importación, exportación; teniendo en cuenta no solo las actividades que se efectúan relacionadas con la manipulación, almacenamiento, sino con las actividades que tienen sobre el transporte anterior y posterior del nodo logístico.

En la zona del Altiplano del Oriente Antioqueño, se vienen asentando y consolidando un sinnúmero de empresas que aprovechan las ventajas comparativas del lugar, la cercanía al Aeropuerto Internacional que lo conecta con diferentes mercados del mundo, una zona franca que otorga prerrogativas arancelarias, tributarias y cambiarias a las empresas que se asientan allí y, una conexión vial que le permite movilizar carga a otras ciudades o a puertos marítimos.
El servicio de contenedores es requerido por las empresas que importan y exportan mercancías vía marítima, los cuales se deben entregar o recoger algunas veces en el municipio de Caldas.

Con este trabajo, se pretende evidenciar, si un puerto seco/patio de contenedores en el Oriente Antioqueño, subregión Altiplano, aportaría a la eficiencia de los procesos de Comercio Exterior.

\section{Marco teórico}

\subsection{Oriente Antioqueño}

El Oriente Antioqueño es una de las nueve subregiones del departamento de Antioquia, conformado por 23 municipios y, dividido en cuatro zonas: Altiplano, Embalses, Bosques y Páramos (Cámara de Comercio del Oriente Antioqueño, 2018), por su ubicación geográfica goza de unas ventajas asociadas a los recursos naturales, lo que aunado a la pujanza de los empresarios y, de sus habitantes se vienen consolidando una serie de actividades relacionadas con el sector agropecuario, agroindustrial, industrial y, de servicios, visionando un futuro promisorio para el turismo.

Según el Banco de la Republica (2005) esta región, ha sufrido cambios gracias a la construcción de carreteras, que la convierten en un punto clave para unir la capital del país con la costa Atlántica y Pacífica, el Oriente y el Occidente y, comunicando a Bogotá y Medellín, mercados de relevancia para la economía de Colombia; un túnel que conecta los dos valles lo que permite reducir significativamente el tiempo de transporte; el Aeropuerto Internacional José María Córdova, con su terminal aérea que sirve al movimiento de pasajeros y carga del Valle de Aburra y, la Zona Franca Rionegro que ofrece 
un sinnúmero de ventajas a los usuarios que se ubican allí; lo que hace atractiva la subregión para el asentamiento de empresas, apuntalando la oferta exportable del departamental, aportando un $18,1 \%$, ubicándose en la segunda posición después del Valle de Aburrá.

Según la Cámara de Comercio del Oriente Antioqueño - CCOA- (2020), en el 2019 se encontraban registradas 19.909 unidades empresariales; de las cuales las personas naturales representan $75 \%$, las personas jurídicas el $25 \%$, en la Zona Altiplano se encuentran asentadas el $79.69 \%$ del tejido empresarial, que corresponden a 9583 empresas, de los cuales, 18.586 son microempresas, 900 pequeña empresa, 328 mediana empresa, 95 gran empresa.

\subsection{Patio de Contenedores}

En 1956, gracias a la utilización del contendor comienza a darse cambios en el manejo de la carga, lo que ayudo a disminuir el tiempo de operación y mano de obra en los puertos. A finales, de la década de los 60 aparecen las terminales de contenedores, como resultado de las operaciones de comercio exterior que se realizaban entre Europa y Estados Unidos donde los portacontenedores se convertían en protagonistas e hicieron que, los puertos se transformaran como dice Muñoz (2008) en puertos de patios destechados y amplios con grandes grúas para manipular la carga; los barcos se transformaron en planchones de mayor capacidad y calado, ayudando a disminuir costos e incrementar la eficiencia de la operación.

En la Política Nacional de Logística (CONPES 3547, 2008) los Patios de Contenedores- Puertos secos:
Se encuentran enmarcados dentro de la concepción de plataformas logísticas especializadas y, se trata de un puerto seco, cuando la terminal intermodal de mercancías esta situada en el interior y conecta a través de la red férrea o carreta con el puerto de origen o destino (pág. 14).

Los puertos secos son establecimientos de comercio, que prestan servicios de recibo, entrega, manipulación, inspección, reparación y limpieza; los cuales se encuentran generalmente fuera de los puertos (pág. 77).

$\mathrm{y}$, para su funcionamiento deben acogerse a la Ley 232 de 1995.

Gómez (2010), aduce que los patios de contenedores se desarrollaron en las ciudades, concebidas de acuerdo a las necesidades que se daban en las urbes y, donde los patios secos se debían adecuar a las demandas de tráfico; pero generaron también, algunos inconvenientes entre la ciudad y los puertos y, como respuesta a esta situación, surge un instrumento de planificación dinámica para tratar de resolver los conflictos que se presentaban en la demanda, competencia inter o intra portuaria, perdida o incorporación de espacios, equipos, problemas de acceso y otros factores que no permitían calcular de manera adecuada la capacidad de estos lugares, mostrando que estos lugares se debían mover a las afueras de la ciudad.

Las empresas que ofrecen los servicios de patios de contenedores, cuentan con espacios reservados para hacer entregas y recibos de estas cajas portátiles a las empresas que las demandan y que se encuentran ubicadas en municipios cercanos; de acuerdo a la Conferencia de las Naciones Unidas sobre el Comercio y Desarrollo -UNCTAD- (2018) no 
todos los contenedores deben ir a un patio de contenedores, algunos van directamente al puerto, esto depende de las políticas de las navieras y del volumen de importaciones y exportaciones que maneje. Para los gobiernos, estos espacios, se convierten en focos de crecimiento económico en los lugares donde se ubican.

\section{Metodología}

Investigación mixta, concluyente, método de investigación analítico. Para la caracterización de los patios de contenedores en Medellín y Caldas Antioquia, se trabajó con 3 de los 4 que se encuentran radicados en el departamento la técnica para recolectar los datos fue una entrevista estructurada a: Contenedores Colombia, ALMAGRARIO y Compañía Colombiana de Logística. Para el análisis de la información fue con la ayuda del software Atlas Ti.

Para evidenciar, si en la región un puerto seco aportaría a la eficiencia de los procesos de comercio exterior, se realizó una investigación cuantitativa, el universo fueron los empresarios que realizan operaciones de importaciones- exportaciones o ambas. En la primera fase del muestreo se hizo una. El tamaño de muestra fue de 63 empresas del Altiplano del Oriente antioqueño, distribuidas de la siguiente manera: 1 Agroindustrial (2\%), 1 Farmacéutica (2\%), 4 del sector alimenticio $(6 \%), \quad 10$ del sector metalmecánico (16\%), 5 de plásticos y derivados de caucho (8\%), 1 del sector maderero (2\%), 12 del sector servicios (19\%), 3 del sector papelero 85\%), 3 minero (5\%), 6 del sector químico (10\%), 4 del sector textil $(6 \%), 1$ de salud y belleza (2\%), 12 del sector agropecuario (19\%). El sistema de muestreo fue aleatorio por etapas. El margen de error del 5\% dentro del límite de confianza de un 95\%. La técnica de recolección de información fue con llamadas de telefónicas a las empresas para determinar cuáles de ellas utilizaban contenedores, luego se les envió la encuesta para a las empresas por medio de google docs y, por último, se realizaron las encuestas a las empresas. Para el análisis se usó la estadística, se hicieron análisis de los resultados y se construyó el informe.

\section{Resultados}

\subsection{Caracterización de los Puertos secos}

Se encontró que en el país hay catorce (14) Patios de contenedores, ubicados en: Cali, Bogotá, Medellín, Cartagena Buenaventura; de los cuales, cuatro (4) están en el departamento de Antioquia, ubicados en Caldas y, son: ALMAGRARIO, SIMARITIM A, Compañía Colombiana de Logística - CCL- y Patio de Contenedores de Colombia-PCC-.

Las tres empresas entrevistadas, poseen patios de contenedores en Bogotá y Medellín, solo dos de ella tienen este tipo de instalaciones en Cartagena, Cali, Barranquilla y Buenaventura y una de ellas en Santa Marta. Estos, Poseen inversiones en otras empresas, para prestar servicios logísticos o de comercio exterior. Los patios de contenedores atienden un sinnúmero de navieras en sus instalaciones y, se destacan: AMERICAN PRESIDENT LINES, CHINA SHIPPING, HAMBURG SUD, HAPAG LLOYD, PACIFIC INTEROCEAN LINES, K LINE, MEDITERRANEAN SHIPPING COMPANY, ZIM LINE, CSAV, EVERGREEN, MITSUI OSK LINES, COSCO, SEA, TEXTAINER, CANONPARCK, LAUCAM, APL, FRONTIER, SAGO, REFRILOGISTIC.

Los reportes que entregan los patios de Contenedores a las navieras son: 
- Reportes diarios de entradas y salidas de contenedores, esto sirve para manejo de inventario.

- Reportes de daños del contenedor

- Consolidados de booking - reservas, permite determinar la disponibilidad de contenedores, que tiene cada patio.

- Presupuestos para reparación de contenedores

- Corrección de errores.

El contenedor que más mueven los patios que se entrevistaron, es el de $40 \mathrm{HC}$ en un $70 \%$ y $30 \%$ los de 20 . Los entrevistados anotan que la distribución viene cambiando en los últimos cinco (5) años, la demanda de los contenedores de 20 Standard disminuye y aumenta la de $40 \mathrm{HC}$ y, se han eliminado los contenedores de 40 Standard.

Ante la pregunta ¿Conoce usted si usuarios de contenedores bien sea importadores o exportadores que estén ubicados en el Oriente Antioqueño? Solo un patio entrevistado, reconoce a algunas empresas que se encuentran ubicadas en el Oriente antioqueño $\mathrm{y}$, que demandan frecuentemente contenedores. Estas empresas son Nacional de Chocolates, Prebel, New Stetic, Griffith de Colombia, Pintuco y Cacharrería mundial. Dos de ellas no saben quién es el cliente, lo conocen por la remisión, ya que el contacto lo tienen directamente con la naviera.

\subsection{Importancia de un Patio Seco}

El servicio de contenedores es requerido por las empresas del Oriente antioqueño que, realizan operaciones de importación y/o exportación, los cuales deben ser entregados o recogidos, la mayoría de las veces en Caldas, municipio de Antioquia. De las 63 empresas a las que se le hizo la encuesta, 10 de ellas $(16 \%)$ requieren el contenedor para operaciones de exportación, 2 (3\%) para la importación en contenedores y 51 empresas (81\%) para procesos de importación y exportación. El transporte internacional que más utilizan las empresas para movilizar la carga en un $45 \%$ el marítimo, $35 \%$ aéreo y el $20 \%$ terrestre.

Entre los productos que importan las empresas de la región, se encuentran: maquinaria, químicos, confecciones, textiles, plásticos, repuestos, material de empaque, motos e insumos. Los contenedores que mayor demandan se encuentran los de 20 y 40 standard y 40 HC. Los lugares más representativos paras para traer mercancías se encuentran China (41\%), Estados Unidos (23\%), Europa (16\%), Suramérica (13\%), Centroamérica $(6 \%)$ y Panamá y Chile en un $1 \%$.

Los productos exportados que se destacan en la región son: flores frescas cortadas, confecciones y textiles, pinturas y barnices, alimentos, papel adhesivo, carbonato de calcio, pinturas en polvo, máquinas $\mathrm{y}$ repuestos, cerámica esmaltadas, aguacates, baldosas entre otros. Los destinos más representativos para las exportaciones son: Suramérica (30\%), Estados Unidos (23\%), Centroamérica (20\%), Europa (12\%), Japón (5\%), Australia (4\%), Panamá $(2 \%)$ y con un $1 \%$ respectivamente China, Chile, Oceanía e Inglaterra.

Las zonas primarias marítimas por donde se realizan las exportaciones son: Cartagena (51\%), Buenaventura (36\%), Santa Marta (10\%), seguidos por Barranquilla (2\%) y Turbo (1\%) y, por donde ingresan las importaciones a Colombia son los puertos de: Buenaventura (47\%), Cartagena (42\%), Santa Marta (5\%) y Barranquilla y Turbo en un $3 \%$ respectivamente. El modo de negociación de contenedores preferido por los encuestados es 
de un $38 \%$ con drop-off sin devolver contenedor a puerto, en un $21 \%$ sin drop-off con devolución en puerto y en un $14 \%$ sin drop-off y sin devolución a puerto.

De acuerdo a la percepción de las personas entrevistadas, consideran que la apertura de un puerto seco en la región, les permitiría optimizar costos, reducir el tiempo de operaciones, eficiencia en las operaciones, y facilitaría procesos de negociación del transporte terrestre; además, un $44 \%$ lo considera como un medio excelente para negociar con las navieras, para un $29 \%$ es bueno, $14 \%$ lo consideran regular, pésimas en un $8 \%$ y deficientes en un $5 \%$ y, permitiría sortear de mejor manera las dificultades con las que se enfrentan en el proceso; también consideran, que las vías de acceso con las que cuenta la región cumplen con las condiciones necesarias para la movilización de estos. Esperan, que, en caso de consolidarse el montaje de una infraestructura de este tipo en alguno de los municipios del Altiplano, debe ofrecer servicios adicionales que generen eficiencias en sus procesos logísticos.

\section{Discusiones}

Las indagaciones realizadas, para dar respuesta, si para los empresarios que realizan operaciones de Comercio Internacional ubicados en el Altiplano, era importante un puerto seco, generó respuestas positivas de los empresarios sobre la necesidad que se tiene de este tipo de operadores en la región, ya que la demanda y oferta de contenedores va en aumento; permitió entender el uso que se le está dando a los contenedores y de cierta forma, los riesgos de daño o contaminación a los que se ven enfrentados, información importante para generar un portafolio de servicios a ofrecer de acuerdo a necesidades específicas de posible usuarios.
De acuerdo a los planteamientos realizados por Gómez (2010) sobre la ubicación de estos patios en las ciudades, los inconvenientes que se generan y, la necesidad de moverlos a las afueras de la ciudad, es necesario que los gobernantes de la región hagan uso de diferentes instrumentos de planificación territorial para evaluar adecuadamente los pros y los contras de albergar en alguno de sus municipios este tipo de instalaciones.

Aunque, estos patios pueden ser uno de los focos de crecimiento económico, se debe considerar que, los patios que prestan los servicios a las empresas del Altiplano, encuentran ubicados en el sur del Valle de Aburra, en el proceso de recogida del contenedor, llevada hasta el Oriente para el proceso de carga y luego esta, debe ser llevada a un Puerto en el Pacifico o en el Atlántico, se generan diversos movimientos que debe hacer un vehículo para movilizar la carga a un lugar de destino, lo que genera para los vehículos un recorrido mayor en kilómetros, aumento en la emisión de $\mathrm{CO} 2$ y deterioro en la calidad de vida de los habitantes de la región.

\section{Conclusiones}

Al analizar el universo de empresas encuestadas pertenecen a 13 sectores diferentes y que ninguno de estos predomina sobre los demás; respecto a la muestra tuvo suficiente amplitud de sectores para ser considerada homogénea en cuanto a la variedad de productos y materias primas que se transportan en los contenedores bien sea de importación o exportación. Es de anotar, que las cantidades de los contenedores puede presentar variaciones por diferentes condiciones económicas, comerciales y políticas que afectan a las empresas. Con el estudio, se obtiene que el $81 \%$ de las 
empresas encuestadas, realizan operaciones tanto de importaciones como de exportaciones, indicando que hay demandantes como oferentes de contenedores hacia los patios. además, se encontró que el movimiento de contenedores en esta zona ha tenido un crecimiento en los últimos 5 años.

Los empresarios del Oriente antioqueño, consideran que la apertura de un patio de contenedores permitirá optimizar costos, tener un menor tiempo de operación y facilitar el transporte desde la empresa hacia el patio y viceversa. También consideran que les permitirá el uso de vías de acceso de manera más adecuada y representa una mejora en la negociación con la naviera.

La región tiene unas características que soportan varias operaciones de comercio exterior, como es un aeropuerto internacional, una zona franca, agencias de carga, bodegas, transportadores, entre otros, falta un servicio de patios de contenedores que le permita tener a la mano lo que necesita, disminuir tiempos y costos

Las certificaciones y los procesos claros ligados a la inspección, registros fotográficos se convierten en un instrumento muy importante en la consolidación de la imagen como prestadores de servicios, lo que sería un gran diferenciador. Otras actividades que se pueden ofrecer desde un patio de contenedores son los centros de distribución, zonas de descongelación, asistencia técnica para refrigerados.

Así mismo se puede observar que el movimiento de componentes o productos intermedios para la elaboración de productos finales, hace parte también de los materiales que ingresan o salen de la región en una clara evidencia de que algunas de estas compañías hacen parte de cadenas globales de valor.

\section{Referencias}

Banco de la República. (2005). La infraestructura del transporte vial y la movilización de carga en Colombia. Recuperado de: https://www.banrep.gov.co/docum/L ectura_finanzas/pdf/DTSER-64.pdf

Cámara de Comercio del oriente Antioqueño (2018). Análisis comercial, Empresarial y regional (ACER). Recuperado de: http:// https://www.ccoa.org.co/camara-yregion/crecimiento-regional.

Cámara de Comercio del Oriente Antioqueño. (2019). Concepto Económico del Oriente Antioqueño. recuperado de: https://www.ccoa.org.co/Portals/0/Le y\%20de\%20transparencia/Concepto $\% 20$ Economico\%202020.pdf

Colombia. Consejo Nacional de Politica Económica y Social - CONPES-. CONPES 3547 (26 de octubre de 2008). Por medio de la cual se dictan normas para el funcionamiento de los establecimientos Comerciales. Recuperado de: https://www.minsalud.gov.co/sites/ri d/Lists/BibliotecaDigital/RIDE/INEC /IGUB/Ley-232-de-1995.pdf

Colombia. Congreso de la Republica. Ley 232 (26 de diciembre 1995). Por medio de la cual se dictan normas para el funcionamiento de los establecimientos Comerciales. Recuperado de: 
https://www.minsalud.gov.co/sites/ri d/Lists/BibliotecaDigital/RIDE/INEC /IGUB/Ley-232-de-1995.pdf

Conferencia de las Naciones Unidas sobre el Comercio y Desarrollo. (2018).

Informe sobre el transporte marítimo. Recuperado de: https://unctad.org/es/PublicationsLibr ary/rmt2018_es.pdf

Gómez, A. (2010).Capacidades de terminales de Contenedores: determinación del área requerida. Recuperado de: http://www.aadip.org.ar/congresoVI/ pdf/paper/dia7/30.Gomez\%20Paz, $\% 2$ 0Maria\%20Alejandra/Gomez\%20Paz _et.al_AADIP2010.pdf

Ministerio de TransporteMINTRANSPORTE-. (2014). Análisis de las condiciones de operación de los patios de contenedores (PC) en Colombia. Recuperado de: file://C:/Users/lceballos/Downloads/ Documento_Patios_de_Contenedores _v4_0_16062014\%20(8).pdf

Muñoz, V. (2008). Optimización de la producción en una terminal marítima de contenedores, umbrales y punto de equilibrio. Recuperado de: https://www.tdx.cat/bitstream/handle/ 10803/7009/01VEmc01de01.pdf?seq uence $=1 \&$ is Allowed $=\mathrm{y}$ 